\title{
Supplementary material: Van der Waals function for molecular mechanics
}

Li Yang ${ }^{1,2,3}$, Lei Sun ${ }^{2}$, and Wei-Qiao Deng ${ }^{1,2,{ }^{*}}$

${ }^{1}$ State Key Laboratory of Molecular Reaction Dynamics, Dalian National Laboratory for Clean

Energy, Dalian Institute of Chemical Physics, Chinese Academy of Sciences, Dalian 116023,

China

${ }^{2}$ Institute of Molecular Sciences and Engineering, Institute of Frontier and Interdisciplinary

Science, Shandong University, Qingdao 266237, China

${ }^{3}$ University of the Chinese Academy of Sciences, Beijing 100039, China

\section{Corresponding Author}

*E-mail: dengwq@sdu.edu.cn 


\section{Section S1: The accuracy of Ab initio calculation}

The stabilization energies, equilibrium distances and energies calculated for $\mathrm{He}-\mathrm{He}$, $\mathrm{He}-\mathrm{Ar}$ and Ar-Ar are shown in table S1. The referenced well parameters $\varepsilon$ and $r_{m}$ are from the semi-empirical potential of Aziz et al. and theoretical values of Anderson with quantum Monte Carlo calculation. ${ }^{1-4}$ A fairly good agreement between the semiempirical and theoretical calculation data obtained with the CCSD(T)/CBS level. The difference between calculated and semi-empirical $r_{\mathrm{m}}$ is smaller than $0.02 \AA$. Percentage error between $0.6 \%$ and $1.7 \%$ are found for well depth, which is very low.

TABLE S1. Comparison of the well locations and well depths of the present calculations with previous experimental and theoretical determinations.

\begin{tabular}{ccccc}
\hline \multirow{2}{*}{ system } & \multicolumn{2}{c}{ Well location $r_{m}(\AA)$} & \multicolumn{2}{c}{ Well depth $\varepsilon(\mathrm{K})$} \\
\cline { 2 - 5 } & present & others & present & others \\
\hline He-He & 2.95 & $2.97^{\mathrm{a}, \mathrm{b}}$ & 10.90 & $10.92^{\mathrm{a}}, 10.98^{\mathrm{b}}$ \\
He-Ar & 3.50 & $3.48^{\mathrm{c}}$ & 30.31 & $29.78^{\mathrm{c}}$ \\
Ar-Ar & 3.75 & $3.76^{\mathrm{d}}$ & 141.73 & $143.36^{\mathrm{d}}$ \\
\hline
\end{tabular}

${ }^{\mathrm{a}}$ Reference 1

${ }^{\mathrm{b}}$ Reference 2

${ }^{\mathrm{c}}$ Reference 3

${ }^{\mathrm{d}}$ Reference 4

In order to explore the relationship between fitting accuracy and QM calculation accuracy, we give the comparisons of QM calculations with different accuracy. Fig. S1 gives the comparison of energies at CCSD(T)/aug-cc-pv5z level and CCSD(T)/CBS level. As shown in Fig. S1, the interaction energies calculated at the CCSD(T)/aug-ccpv5z level are lower than energies calculated at the higher level CCSD(T)/CBS near the equilibrium distance. The difference between the energies calculated at two accuracy levels far away from the equilibrium distance is not significant. The Fig. S2 shows the comparisons of the QM and fitted interaction energies. Exp-PE potential shows the best performance in fitting the QM energies with the two calculation levels. As shown in Figs. 3a and 3b, the QM method level nearly has little effect on the fitting accuracy for Exp-PE and LJ 12-6 potentials. 


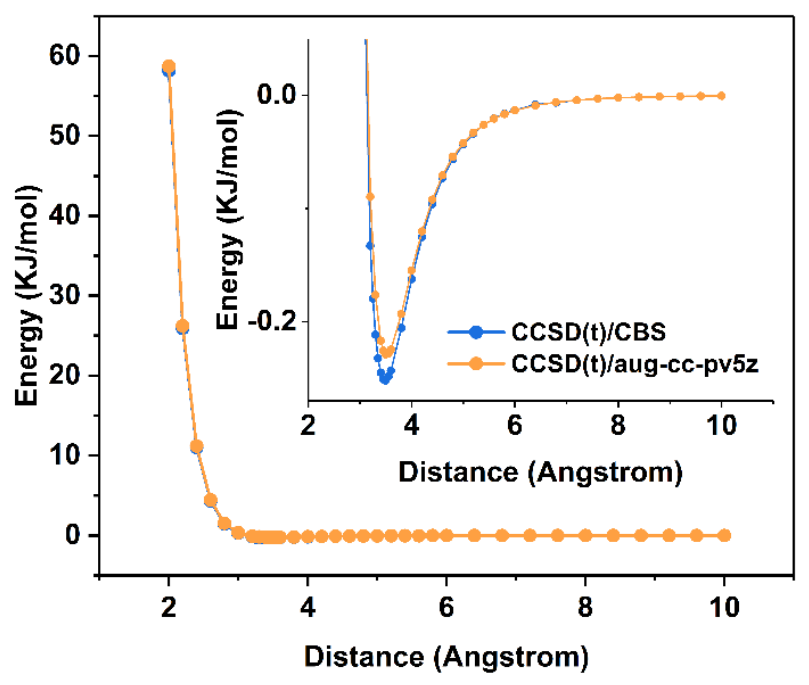

FIG. S1. Comparison of the QM calculations with different accuracy for He-Ar system.

(a)

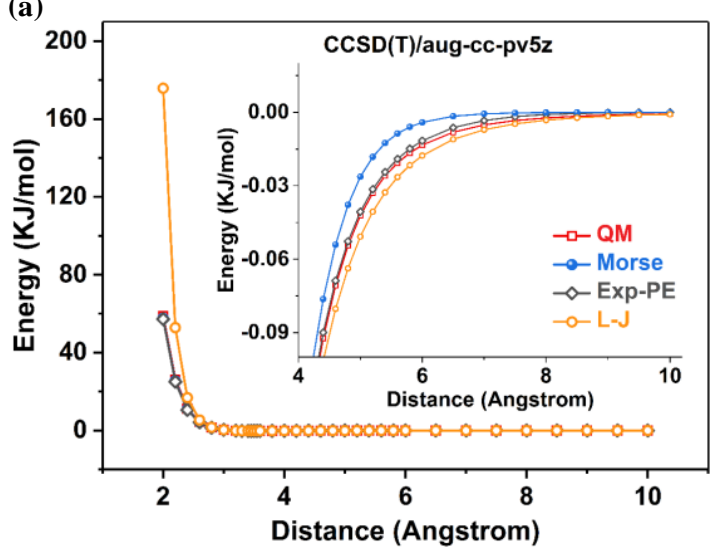

(b)

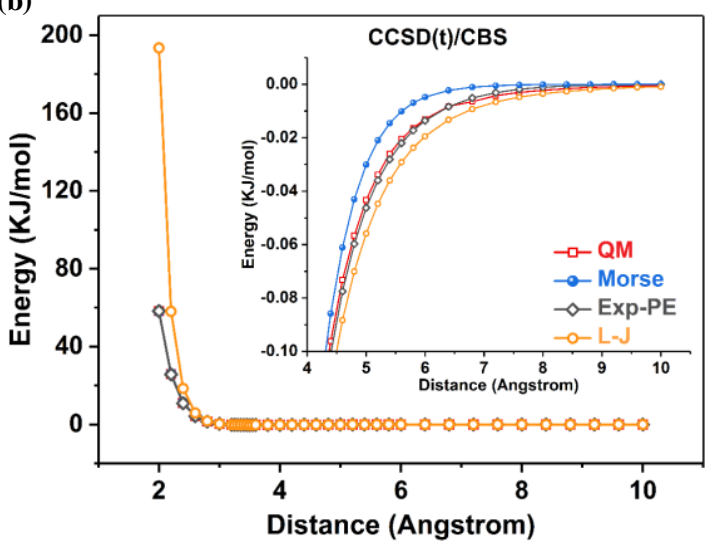

FIG S2. Comparisons of the QM and fitted interaction energies: QM energies are calculated at the CCSD(T)/aug-cc-pv5z level, (a); at the CCSD(T)/CBS, (b).
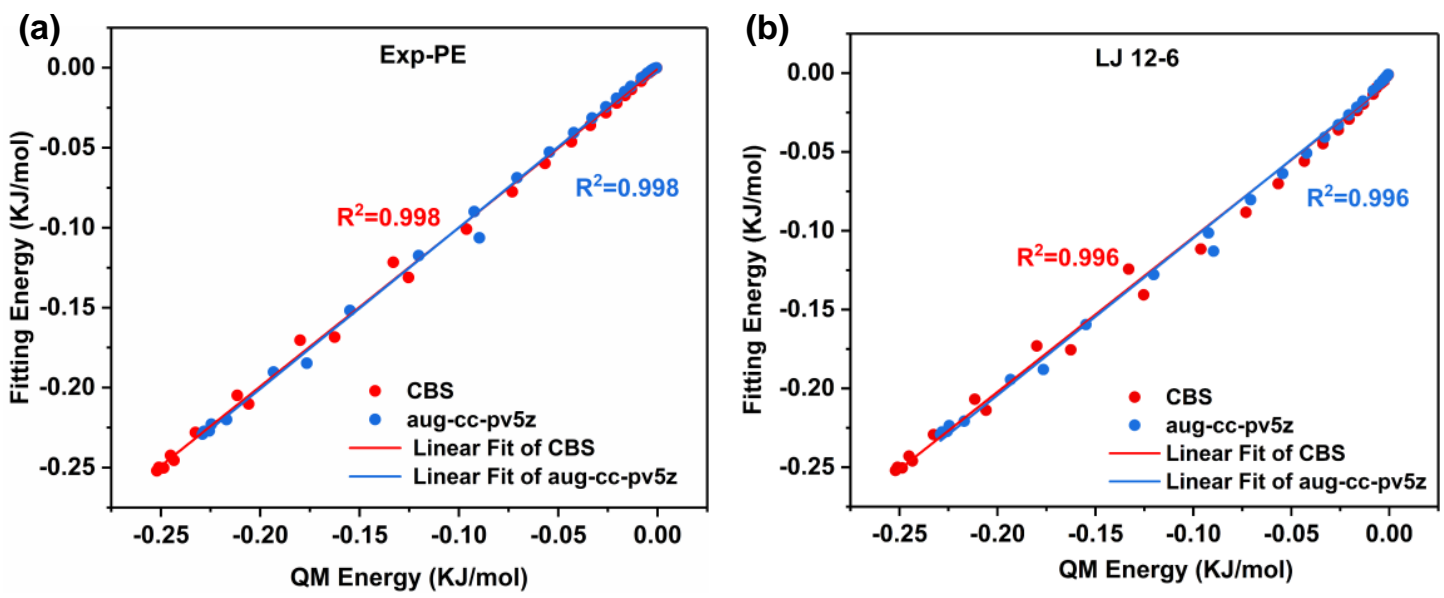

FIG S3. Comparison of the accuracy in fitting to the energies of He-Ar at different calculation levels. 
TABLE S2. Parameters of Potential Functions Developed To Fit the CCSD(T) Calculations.

\begin{tabular}{|c|c|c|c|c|c|}
\hline \multirow{2}{*}{ Systems } & \multicolumn{2}{|c|}{ LJ 12-6/ Buf-14-7 } & Exp-6 & Morse & Exp-PE \\
\hline & $\varepsilon\left(\mathrm{KJ} \mathrm{mol}^{-1}\right)$ & $r_{m}(\AA)$ & & $\alpha$ & \\
\hline $\mathrm{He}-\mathrm{He}^{\mathrm{a}}$ & 0.091 & 2.95 & 13.98 & 13.00 & 13.13 \\
\hline $\mathrm{He}-\mathrm{Ar}^{\mathrm{a}}$ & 0.252 & 3.50 & 13.78 & 13.00 & 13.08 \\
\hline $\mathrm{Ar}-\mathrm{Ar}^{\mathrm{a}}$ & 1.178 & 3.75 & 13.99 & 13.07 & 13.18 \\
\hline $\mathrm{Xe}-\mathrm{Ar} \mathrm{r}^{\mathrm{b}}$ & 1.542 & 4.10 & 14.00 & 12.90 & 13.08 \\
\hline
\end{tabular}


Section S2: Benchmark the performance for different potential functions

TABLE S3. Potential function forms

\section{Potential forms}

LJ 12-6

$$
U(r)=\varepsilon\left[\left(\frac{r_{m}}{r}\right)^{12}-2\left(\frac{r_{m}}{r}\right)^{6}\right]
$$

Exp-6

$$
U(r)=\varepsilon\left[\frac{6}{\alpha-6} \exp \left(\alpha\left(1-\frac{r_{m}}{r}\right)\right)-\frac{\alpha}{\alpha-6}\left(\frac{r_{m}}{r}\right)^{6}\right]
$$

Morse

$$
U(r)=\varepsilon\left[\exp \left(\alpha\left(1-\frac{r}{r_{\mathrm{m}}}\right)\right)-2 \exp \left(\frac{\alpha}{2}\left(1-\frac{r}{r_{\mathrm{m}}}\right)\right)\right]
$$

Buf-14-7

$$
U(r)=\varepsilon\left(\frac{1+\delta}{\frac{r}{r_{m}}+\delta}\right)^{7}\left(\frac{1+\gamma}{\left(\frac{r}{r_{m}}\right)^{7}+\gamma}-2\right)
$$

We only considered one relatively lower-energy geometrical configuration for $\mathrm{H}_{2}$ $\mathrm{H}_{2}, \mathrm{~N}_{2}-\mathrm{N}_{2}, \mathrm{CH}_{4}-\mathrm{CH}_{4}, \mathrm{O}_{2}-\mathrm{O}_{2}$, LiH-He and HF-Kr dimers. For $\mathrm{H}_{2}-\mathrm{H}_{2}, \mathrm{~N}_{2}-\mathrm{N}_{2}, \mathrm{O}_{2}-\mathrm{O}_{2}, \mathrm{LiH}-$ $\mathrm{He}$, and HF-Kr dimers, the distance between two monomers is characterized by the distance between the center of mass. While for $\mathrm{CH}_{4}-\mathrm{CH}_{4}$ the distance is the separation between C-C atoms. Fitting parameters for different kinds of potential functions were listed in table 1.

TABLE S4. Parameters of Exp-PE function fitting the AFM measurements.

\begin{tabular}{cccc}
\hline \multirow{2}{*}{ Systems } & \multicolumn{3}{c}{ Exp-PE } \\
\cline { 2 - 4 } & $\varepsilon(\mathrm{mev})$ & $r_{m}(\mathrm{pm})$ & $\alpha$ \\
\hline $\mathrm{Xe} \mathrm{Xe}^{\mathrm{a}}$ & 36.3 & 475.2 & 11.82 \\
$\mathrm{Xe}-\mathrm{Ar}^{\mathrm{a}}$ & 18.0 & 451.0 & 14.18 \\
$\mathrm{Xe-Kr^{ \textrm {a } }}$ & 26.1 & 462.9 & 13.11 \\
\hline
\end{tabular}




\section{Section S3: Integral method of second virial ccoefficient}

According to classical statistical mechanics, the second virial coefficient $B(T)$ of gases whose molecules interact in a central force law can be expressed as :

$$
B(T)=-\frac{2 \pi N}{3 k T} \int_{0}^{\infty} r^{3} \frac{d \phi(r)}{d r} e^{-\phi(r) / k T} d r
$$

$\phi(r)$ is the potential energy of interaction between pairs of molecules. $B(T)$ can be written as the function of the reduced second virial coefficient:

$$
\begin{gathered}
B(T)=(2 / 3) \pi N r_{m}^{3} B^{*}\left(C, T^{*}\right) \\
B^{*}\left(T^{*}\right)=-3 \int_{0}^{\infty} r^{* 2}\left[1-e^{-\phi^{*}\left(r^{*}\right) / T^{*}}\right] d r^{*}
\end{gathered}
$$

Where $N$ is the Avogadro number, $C$ is the parameter in Morse function. $B^{*}$, $T^{*}$, and $r^{*}$ are the reduced second virial coefficients, temperature and the distance between two atoms, respectively. $T^{*}=k T / \varepsilon$ and $r^{*}=r / r_{m}$, where $T$ is the temperature, $k$ is the Boltzmann constant, $\varepsilon$ is the well depth and $r_{m}$ is the equilibrium position. As the value of the exponential term decay rapidly with $r^{*}$ increasing, we divided the reduced second virial coefficient into two parts: integration from $r^{*}=0$ to $r^{*}=1$ and from $r^{*}=1$ to $r^{*}=20$. Integrals for each subinterval were evaluated with composite Cotes' formula involving a large number of abscissas (n1=100000, n2=1000000).

Second virial coefficient for binary mixture:

$$
\begin{gathered}
B_{i j}=\frac{2}{3} \pi N_{A} \sigma_{i j}^{3} B_{i j}^{*} \\
B=x_{1}^{2} B_{11}+2 x_{1} x_{2} B_{12}+x_{2}^{2} B_{22}
\end{gathered}
$$




\section{Section S4: Details of derivation process}

In order to explore the exact potential form of two body interaction in the middle and long-range, we re-derived the function. For simplicity, we shall consider the interaction of two neutral hydrogen atom (A) and a proton (B) in the ground state $\left(\mathrm{H}_{2}\right)$ (figure S8). We write the Hamilton of the system as

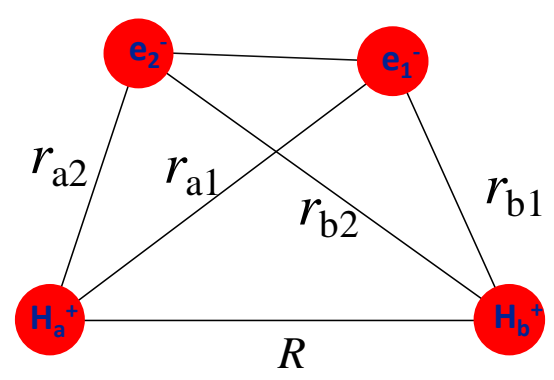

FIG. S4. Schematic illustration of the $\mathrm{H}_{2}$ system. $\mathrm{H}_{\mathrm{a}}{ }^{+}$and $\mathrm{H}_{\mathrm{b}}{ }^{+}$are two identicalhydrogen cores.

$$
\hat{H}=-\frac{1}{2}\left(\nabla_{1}^{2}+\nabla_{2}^{2}\right)+\frac{1}{r_{12}}-\left(\frac{1}{r_{a 1}}+\frac{1}{r_{a 2}}+\frac{1}{r_{b 1}}+\frac{1}{r_{b 2}}\right)+\frac{1}{R}
$$

Here the "1 and 2" refers to electron one and two, "a and b" refers to two $\mathrm{H}$ nuclei, $R$ and $r_{12}$ are the distances between two $\mathrm{H}$ nuclei and two electrons, $r_{\mathrm{a} 1}, r_{\mathrm{a} 2}, r_{\mathrm{b} 1}$ and $r_{\mathrm{b} 2}$ are the distances between $\mathrm{H}$ nuclei and electrons. Suppose the system be in the ground state. The atomic hydrogen orbitals can be written as

$$
\psi(r)=\frac{\lambda^{3 / 2}}{\sqrt{\pi}} \exp [-\lambda r]
$$

$\lambda$ can be treated as an effective charge. The ground state wave function for the $\mathrm{H}_{2}$ is

$$
\Psi_{\mathrm{s}}=\left[\psi\left(r_{a 1}\right) \psi\left(r_{b 2}\right)+\psi\left(r_{a 2}\right) \psi\left(r_{b 1}\right)\right]
$$

The corresponding energies in terms of ground state wave function is

$$
E_{s}=\frac{1}{R}+\frac{2\left(A+A^{\prime} s\right)}{1+s^{2}}-\frac{2(\kappa+\varepsilon s)-\left(\kappa^{\prime}+\varepsilon^{\prime}\right)}{1+s^{2}},
$$

where $s$ is the overlap integral, $\kappa$ and $\kappa^{\prime}$ is the coulomb integral, A is correspond to the ground state energy of one $\mathrm{H}$ atom. A, $\kappa$ and $\kappa^{\prime}$ are independent on $s$, and can be understood by classical images. $\varepsilon, \varepsilon^{\prime}$ and $A^{\prime}$ are the energy caused by 
exact quantum effects (exchange effects) and has no corresponding classical images. Let $\rho=\lambda R$, then

$$
\begin{gathered}
s=\int d \tau_{1} \psi^{*}\left(r_{a 1}\right) \psi\left(r_{b 1}\right)=\left(1+\rho+\frac{1}{3} \rho^{2}\right) \exp (-\rho), \\
\kappa=\int d \tau_{1}\left|\psi\left(r_{a 1}\right)\right|^{2} / r_{b 1}=\frac{\lambda}{\rho}\{1-(1+\rho) \exp (-2 \rho)\} \\
\varepsilon=\int d \tau_{1} \psi^{*}\left(r_{a 1}\right) \psi\left(r_{b 1}\right) / r_{a 1}=\lambda\left\{s(\rho)-1 / 3 \rho^{2} \exp (-\rho)\right\} \\
A=\int d \tau_{1} \psi^{*}\left(r_{a 1}\right) F\left(r_{a 1}\right)=-\lambda^{3} / 2+\lambda(\lambda-1), \\
A^{\prime}=\int d \tau_{1} \psi^{*}\left(r_{a 1}\right) F\left(r_{b 1}\right)=-\lambda^{2} s / 2+\varepsilon(\lambda-1), \\
\quad F(r)=\left(-1 / 2 \nabla^{2}-1 / r\right) \psi(r) \\
\varphi(\rho)=s(\rho)^{2}(\ln \rho+C)-s(-\rho)^{2} E_{1}(4 \rho)+2 s(\rho) s(-\rho) E_{1}(2 \rho) \\
\kappa^{\prime}=\iint d \tau_{1} d \tau_{2}\left|\psi\left(r_{a 1}\right)\right|^{2}\left|\psi\left(r_{b 2}\right)\right|^{2} / r_{12}, \\
\varepsilon^{\prime}=\iint d \tau_{1} d \tau_{2} \psi^{*}\left(r_{a 1}\right) \psi\left(r_{b 1}\right) \psi^{*}\left(r_{b 2}\right) \psi\left(r_{a 2}\right) / r_{12} \\
=\lambda\left\{\left(5 / 8-23 / 20 \rho-3 / 5 \rho^{2}-1 / 15 \rho^{3}\right) \exp (-2 \rho)+6 / 5 \varphi(\rho) / \rho\right\} \\
E_{\rho}^{\infty} \frac{1}{t} \exp (-t) d t .
\end{gathered}
$$

Where $\mathrm{C}=0.57722$, is the Euler constant. Assuming the exponential integral $E_{1}(\rho)$ and the overlap integral $s^{2}$ are small enough to be negligible, the $E_{\mathrm{s}}$ can be expressed as follows:

$$
E_{s}=2 A+\left(C_{-1} \rho^{-1}+C_{0} \rho^{0}+C_{1} \rho^{1}+C_{2} \rho^{2}+C_{3} \rho^{3}+C_{4} \rho^{4}\right) \exp (-2 \rho) .
$$

In order to explore the exact potential form of two body interaction in the middle and long-range, we also re-derived the function for $\mathrm{H}_{2}{ }^{+}$system using perturbation method. For simplicity, we shall consider the interaction of a neutral hydrogen atom (A) and a proton (B) in the ground state $\left(\mathrm{H}_{2}^{+}\right)$(Fig. S5). We write the Hamilton of the system as 


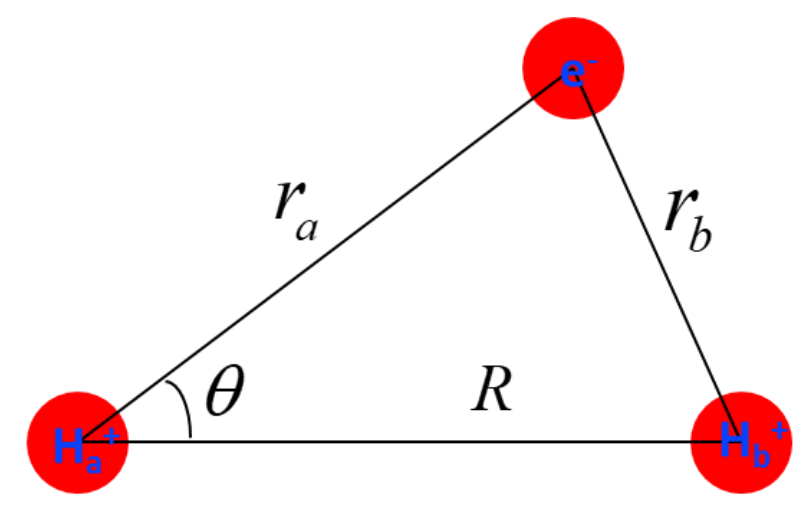

FIG. S5. Schematic illustration of the $\mathrm{H}_{2}{ }^{+}$system. $\mathrm{H}_{\mathrm{a}}{ }^{+}$and $\mathrm{H}_{\mathrm{b}}{ }^{+}$are two identicalhydrogen cores.

$$
\begin{aligned}
& H=H_{0}+H^{\prime}, \\
& H_{0}=-\frac{1}{2} \nabla^{2}-\frac{1}{r_{a}}, \\
& H^{\prime}=\frac{1}{R}-\frac{1}{r_{b}},
\end{aligned}
$$

where $H_{0}$ is unperturbed Hamiltonian, $H^{\prime}$ can be regard as a weak physical disturbance. Suppose the hydrogen atom be in the ground state. Because the two atoms are far away from each other, their electron wave function overlaps very little, and the exchange effects can be omitted. Thus the zero-order wave function of the system can be expressed as $\Psi_{0}=1 s$. The radial part of the Slater orbital function ${ }^{5} 1 s$ is $e^{-r} / \sqrt{\pi}$. To the second order, the energy correction reads

$$
E^{(2)}=\sum_{k \neq 0} \frac{\left|\left\langle 0\left|H^{\prime}\right| k\right\rangle\right|^{2}}{E_{0}^{0}-E_{k}^{0}} .
$$

An approximate evaluation of Eq. (23) is obtained by following Unsold's ${ }^{6}$ procedure:

$$
E^{(2)}=-\frac{1}{\varepsilon}\left\{\left\langle 0\left|H^{\prime 2}\right| 0\right\rangle-\left\langle 0\left|H^{\prime}\right| 0\right\rangle^{2}\right\} .
$$


Since Slater orbital functions $1 s$ are even and $H^{\prime}$ is an odd parity operator, the first-order energy $\left\langle 0\left|H^{\prime}\right| 0\right\rangle$ is zero. Some authors ${ }^{7-8}$ expanded $H^{\prime}$ in Legendre polynomials:

$$
H^{\prime}=-\frac{2}{R} \sum_{n=1}^{\infty}\left(r_{b} / R\right)^{n} P_{n}\left(\cos \theta_{b}\right)
$$

With the use of the ground-state wave function for hydrogen and the orthogonality property of the Legendre polynomials, they obtain the asymptotic series:

$$
E^{(2)}=-\frac{1}{\varepsilon} \sum_{n=1}^{\infty} \frac{(2 n+2) !}{2^{2 n+1}(2 n+1)} R^{-(2 n+2)} .
$$

This approximation seems to be somewhat crude, as Eq. (11) is valid only for $r_{b}<R$, but Eq. (25) is obtained by expanding the integral range from $0-R$ to $0-\infty$. In order to explore the real expression for $E^{(2)}$, we re-calculated Eq. (23) using the exact expansion form of $H^{\prime}$.

$$
\begin{aligned}
& \left\langle 0\left|H^{\prime 2}\right| 0\right\rangle=\frac{1}{R^{2}}-\frac{2}{R}\left\langle 0\left|r_{b}^{-1}\right| 0\right\rangle+\left\langle 0\left|r_{b}^{-2}\right| 0\right\rangle \\
& \left.\qquad 0\left|r_{b}^{-1}\right| 0\right\rangle=\frac{1}{R}\left[1-(1+R) e^{-2 R}\right] \\
& \left\langle 0\left|r_{b}^{-2}\right| 0\right\rangle=4 \sum_{n=0}^{\infty} \frac{1}{2 n+1}\left[\frac{1}{R^{2 n+2}} \int_{0}^{R} r^{2 n+2} e^{-2 r} d r+R^{2 n} \int_{R}^{\infty} r^{-2 n} e^{-2 r} d r\right] \\
& =4 \sum_{n=0}^{\infty} M_{n} \\
& M_{n}=\frac{1}{4 R^{2}}\left[1-(1+2 R) e^{-2 R}\right], \\
& =\frac{1}{2 n+1}\left[\frac{\mathrm{A}_{2 n+2}^{2 n+2}}{2^{2 n+3}} R^{-2 n-2}-\sum_{l=0}^{2 n+2} \frac{\mathrm{A}_{2 n+2}^{l} R^{-l}}{2^{l+1}} e^{-2 R}+\right. \\
& \left.\sum_{l=0}^{2 n-2} \frac{R^{l+1}}{\mathrm{~A}_{2 n-1}^{l+1}(-2)^{l}} e^{-2 R}+\frac{R^{2 n}}{(-2)^{2 n-1}} \int_{R}^{\infty} r^{-1} e^{-2 r} d r\right], \quad n \geq 1
\end{aligned}
$$

Assuming that the terms involving the exponential integral are small, hence 


$$
E^{(2)}=-\frac{1}{\varepsilon}\left(\sum_{n=1}^{\infty} \sum_{k=-2 n-1}^{2 n-1} C_{k} R^{k} e^{-2 R}+\sum_{n=1}^{\infty}\left(1-e^{-2 R}\right) \frac{(2 n+2) !}{2^{2 n+1}} R^{-2 n-2}\right) .
$$




\section{Reference}

1. Aziz, R. A.; Slaman, M. J. An examination of abinitio results for the helium potential energy curve. J. Chem. Phys. 1991, 94, 8047-8053.

2. Anderson, J. B. An exact quantum Monte Carlo calculation of the helium-helium intermolecular potential. II. J. Chem. Phys. 2001, 115, 4546-4548.

3. Aziz, R. A. In Inert Gases: Potentials, Dynamics and Energy Transfer in Doped Crystals. Springer: Berlin,1984.

4. Aziz, R. A. A highly accurate interatomic potential for argon. J. Chem. Phys. 1993, 99, 4518-4525.

5. Roothaan, C. C. J. A study of two-center integrals useful in calculations on molecular structure. I. J. Chem. Phys. 1951, 19, 1445-1458.

6. Unsold, A. Quantentheorie des Wasserstoffmolekülions und der Born-Landéschen Abstoßungskräfte. Z. Physik 1927, 43, 563.

7. Brooks, F. C. Convergence of intermolecular force series. Phys. Rev. 1952, 86, 92.

8. Dalgarno, A. J.; Lewis, T. The representation of long range forces by series expansions I: The divergence of the series II: The complete perturbation calculation of long range forces. Proc. Phys. Soc. A 1956, 69, 57. 